\title{
Lean management-Reformen als Ansatz zur Überwindung struktureller Dysfunktionalität öffentlicher Verwaltungen in Afrika südlich der Sahara - Überlegungen am Beispiel Benins und Ugandas ${ }^{1}$
}

\author{
Von Hildegard Lingnau
}

\section{Problemauf riß}

In vielen Entwicklungsländern sind Staat und Verwaltung nicht, wie lange erhofft, Träger und Förderer von Entwicklung, sondern stellen vielmehr ein Entwicklungshemmnis dar. ${ }^{2}$ Vor allem in Ländern Afrikas südlich der Sahara treten Staatsapparate oftmals als kleptokratische Kommandostaaten in Erscheinung. Dieser Sachverhalt ist historisch und politisch bedingt. Despotische Herrschaftsformen aus vorkolonialer Zeit wurden während der Kolonialzeit um zentralstaatliche Herrschafts- und Ausbeutungsapparate ergänzt. Positive (Selbst-)Verwaltungserfahrungen konnten innerhalb dieser Strukturen nicht gemacht werden. Nach der Unabhängigkeit wurden in fast allen Ländern Afrikas südlich der Sahara und unabhängig von deren jeweiliger politischer Ausrichtung etatistische Entwicklungsmodelle verfolgt. Aus entwicklungspolitischer Sicht sind diese als weitgehend gescheitert anzusehen, weil sie nicht zur Verfolgung und Umsetzung entwicklungsorientierter Politiken im Interesse des Gemeinwohls geführt haben, sondern vielmehr den herrschenden Eliten die Sicherung von Renten und Privilegien - nicht zuletzt im Rahmen der Entwicklungszusammenarbeit - ermöglichten.

Im Rahmen von Strukturanpassungspolitiken sind viele Regierungen in Ländern Afrikas südlich der Sahara, deren Staatsbudgets zu einem Großteil aus Mitteln der Entwicklungszusammenarbeit finanziert werden, unter massiven Druck geraten. Dieser Druck aber blieb weitgehend auf austeritätspolitische Maßnahmen (Haushaltssanierung durch Ausgabenkürzungen und Erhöhung von Einnahmen) begrenzt. Die effektive Reformierung von Makround Sektorpolitiken wurde vernachlässigt. Dies hat in vielen Fällen dazu geführt, daß Rahmenbedingungen nach wie vor wenig entwicklungsförderlich sind und Macht- und Vertei-

Der vorliegende Artikel stellt eine Zusammenfassung des Buches "Lean management als Konzept zur Reform öffentlicher Verwaltungen in Afrika südlich der Sahara - Schlußfolgerungen aus den 2 Verwaltungsreformen Benins und Ugandas" dar, das 1996 im Weltforum-Verlag erschienen ist.

Vgl. A. Stockmayer, Staatliche Verwaltung - Hemmschuh für die Entwicklung? Die Qualität der Verwaltungskultur prägt Staat und Wirtschaft, in: gtz-info, H. 3, 1991. 
lungskämpfe durch Steuererhöhungen, Reduktion von Bildungs-, Gesundheits- und Sozialausgaben etc. vor allem zu Lasten der Mehrheit der Bevölkerung verschärft wurden.

Im Bereich der öffentlichen Verwaltung kommt die enge und unpolitische Fokussierung von Strukturanpassungs- und Entwicklungspolitiken darin zum Ausdruck, daß Staat und Verwaltung lediglich als zusätzlicher Sektor oder, sektorübergreifend, als neutrale, interessenlose Akteure wahrgenommen werden, statt als das, was sie vor allem sind: Schlüsselfaktor und Rahmenbedingung politischer und wirtschaftlicher Entwicklung. Öffentliche Verwaltungen in Afrika südlich der Sahara, die als erdrückende Staatsapparate große Teile der Bevölkerung im Dienst kleptokratischer Eliten bevormunden und ausbeuten, müssen daher dringend reformiert und zu effizienten Akteuren im Dienste der Allgemeinheit umgestaltet (nicht jedoch zwangsläufig abgebaut) werden. Nur so kann die Regierungsführung ("good governance") verbessert und die Abwärtsspirale einander bedingender wirtschaftlicher und politischer Unterentwicklung durchbrochen werden.

Es stellt sich somit die Frage, wie öf fentliche Verwaltungen in Afrika südlich der Sahara so reformiert werden können, daß sie Entwicklungsprozesse nicht behindern, sondern möglichst effizient und kompetent fördern. Im folgenden wird versucht, dazu einen Beitrag zu leisten, indem die Reformbemühungen zweier afrikanischer Länder vor dem Hintergrund des aktuellen Reformkonzeptes "lean management" analysiert werden.

\section{Öffentliche Verwaltung in Afrika südlich der Sahara}

Öffentliche Verwaltungen im Sinne einer neutralen und separaten Gewalt im Rahmen eines ausdifferenzierten politischen Systems hat es im vorkolonialen Afrika nicht gegeben. Die Gesellschaftsstruktur des weitgehend staatenlosen vorkolonialen Afrikas war durch eine Vielzahl unterschiedlichster Fürstentümer, Königreiche und akephaler Gesellschaften geprägt. Bei aller Unterschiedlichkeit von Verwaltungsstrukturen der vielen verschiedenen Gesellschaften im vorkolonialen Afrika läßt sich feststellen, daß es sich in den meisten Fällen um despotische und patriarchalische Systeme gehandelt hat, die denkbar ungeeignet waren und sind, den heute allgemeingültigen Anforderungen an öffentliche Verwaltungen zu entsprechen, die sich in den letzten Jahrzehnten und Jahrhunderten im Zuge von Aufklärung und Demokratisierung herausgebildet haben. "Die despotischen Systeme in weiten Teilen der früheren "Dritten Welt" sind nicht in erster Linie das Resultat westlicher Manipulationen, sondern gründen sich auf Traditionen, die schon bestanden haben, bevor die ersten Spanier, Portugiesen, Niederländer, Engländer und Franzosen das Land betreten haben." ${ }^{3}$ Auch Brett rät massiv davon ab, bei der Suche nach alternativen Verwaltungs-

U. Menzel, Das Ende der Dritten Welt und das Scheitern der großen Theorie, Frankfurt a.M. 1992, S. 220. 
systemen auf vorkoloniale Strukturen zu rekurrieren. Er konstatiert "inappropriateness of traditional centralized state structures as service delivery systems in situations characterized by limited information, poor communications, scarce managerial skills, hierarchical and patriarchal social structures, and the lack of effective mechanisms for establishing a basis for trust between individuals and agencies." 4

Während der Kolonialzeit haben die Kolonialmächte mehr oder weniger weitgehend und mehr oder weniger erfolgreich versucht, den Kolonien ihre Verwaltungsstrukturen aufzuoktroyieren. ${ }^{5}$ Im Unterschied zu den Mutterländern dienten Staat und Verwaltung allerdings weniger dazu, Rechtsstaatlichkeit herzustellen, als die Unterwerfung und Ausbeutung af rikanischer Völker und Gesellschaften zu organisieren. ${ }^{6}$

Die postkolonialen Staaten und Bürokratien entstanden nicht aus der Ausdifferenzierung der Gesellschaft wie in Europa, sondern aus der Übernahme kolonialer Strukturen und deren willkürlicher Instrumentalisierung. Die neuen Regierungen der unabhängig gewordenen Staaten kopierten zumeist die Verfassungen ihrer früheren Kolonialmächte ${ }^{7}$ und praktizierten Staat und Verwaltung so, wie sie es aus der Kolonialzeit kannten, als autoritäre bis tyrannische ${ }^{8}$ Zwangssysteme zur Unterwerfung der unterschiedlichen ethnischen und gesellschaftlichen Gruppierungen, ${ }^{9}$ mit einer Obrigkeitsverwaltung, der es in erster Linie auf die Bewahrung und Konsolidierung bestehender Machtverhältnisse ankam. ${ }^{10}$ In den meisten Ländern Afrikas südlich der Sahara entwickelten sich diktatorische Systeme mit

4

Vgl. E.A. Brett, Providing for the Rural Poor: Institutional Decay and Transformation in Uganda, IDS Research Report 23, S. i, Brighton 1992

5

Vgl. F. Ansprenger, Politische Geschichte Afrikas im 20. Jahrhundert, München 1992, Kap. 3.

6 Vgl. G. Mutahaba / R. Baguma / M. Halfani, Vitalizing African Public Administration for Recovery and Development, West Hartford/Connecticut 1993.

Vgl. F. Ansprenger, Politische Geschichte..., a.a.O. und D. Kohnert, Perspektiven der Liberalisierung von Wirtschaft und Gesellschaft in den neuen Demokratien Schwarzafrikas, Hamburg 1995.

"Was wir (Briten und Franzosen zumeist) Afrika im Augenblick der staatlichen Unabhängigkeit an Politikmustern, an Verfassungstexten hinterlassen haben, taugte nichts, weil die 'Verfassungswirklichkeit' unserer eigenen Kolonialregime in Afrika bis fünf Minuten vor zwölf nicht demokratisch war, sondern autoritär bis tyrannisch." (F. Ansprenger, Politische Geschichte... a.a.O., S. 175)

Vgl. A. Mbembe, Pouvoir, violence et accumulation, in: J.F. Bajart / A. Mbembe / C. Toulabor, La politique par le bas en Afrique noire - Contributions à une problématique de la démocratie, Paris 1992 und P.J. Hountondji, The Crisis of the State in Africa, in: C. Auroi (Hrsg.), The Role of the State in Development Processes, London 1992.

10 Vgl. M. Bohnet, Förderung der Verwaltung in Entwicklungsländern: Rückblick und Perspektiven für die 90er Jahre, in: K. Simon / A. Stockmayer / H. Fuhr (Hrsg.), Subsidiarität in der Entwicklungszusammenarbeit: Dezentralisierung und Verwaltungsreform zwischen Strukturanpassung und Selbsthilfe, Baden-Baden 1993. 
zentralistischen Strukturen, ${ }^{11}$ deren Legitimität "in vielen Fällen das pure Geschenk der Kolonialherren", darüber hinaus aber weder demokratisch noch funktional gegeben war. ${ }^{12}$ Mit der Begründung, der Staat sei der einzig mögliche Entwicklungsakteur, wurde ein Großteil der Wirtschaft in vielen Ländern Afrikas südlich der Sahara verstaatlicht und die öffentliche Verwaltung ausgebaut ("interventionist state"). ${ }^{13}$ Der öffentliche Dienst und verstaatlichte Unternehmen wurden als Instrument gesellschaftlicher Befriedung angesichts fehlender Arbeitsplätze, aber auch zur Versorgung der jeweiligen Klientel instrumentalisiert. Im Interesse einer breitenwirksamen und nachfragefördernden Wohlfahrtspolitik erschien dieses Vorgehen lange Zeit durchaus akzeptabel. Heute allerdings muß man feststellen, daß Staat und Verwaltung in vielen Ländern Afrikas südlich der Sahara vor allem dort präsent sind, wo Ressourcen verteilt und Einnahmen (Abgaben, Abschöpfungen, Renten etc.) erzielt werden, d.h. im Bereich von Hoheits- und Planungsverwaltungen, konkret beim Zoll, bei staatlichen marketing boards und allen anderen mit Produktion, Handel und Entwicklungszusammenarbeit befaßten Unternehmen und Institutionen. Staat und Verwaltungen treten hier weniger als Förderer und Dienstleister in Erscheinung, sondern eher als dirigistischer und rentenorientierter Obrigkeitsstaat, der Bauern und Bäuerinnen nicht nur von oft inkompetenten Beratern des Landwirtschaftsministeriums vorschreiben läßt, wann sie beispielsweise zu säen haben, ${ }^{14}$ sondern sich auch mittels Steuern und Abgaben, Vermarktungsmonopole und staatlich festgesetzter niedriger Ankaufpreise auf Kosten großer Teile der Bevölkerung bereichert. ${ }^{15}$ Wo es staatliche Leistungen zu erbringen gilt, so z.B. im Bildungs- und Gesundheitswesen - von "Sozialpolitik" ganz zu schweigen - sind Staat und Verwaltung kaum präsent. Der Großteil der Bevölkerung der Länder Afrikas südlich der Sahara versucht daher verständlicherweise, so wenig wie möglich mit staatlichen Stellen in Kontakt zu kommen.

Zusammenfassend läßt sich festhalten, daß sich in den meisten Ländern Afrikas südlich der Sahara aufgrund fehlender afrikanischer Verwaltungstraditionen und mißbräuchlich instrumentalisierter "moderner" Verwaltungsstrukturen kein neutrales und dienstleistungsorientiertes Staats- und Verwaltungsverständnis herausgebildet hat. Vielmehr haben sich vorkoloniale despotische Herrschafts- und Verwaltungsstrukturen mit den zwangsstaatlichen der Kolonialverwaltung vermischt, die in der postkolonialen Zeit dank klientelistischer Praktiken und umfassender und weitgehend vorbehaltsloser Außenfinanzierung

Vgl. F. Ansprenger, Politische Geschichte..., a.a.O. und J. Healy / M. Robinson, Democracy, Governance and Economic Policy - Subsaharan Africa in Comparative Perspective, London 1992. Vgl. U. Menzel, Das Ende..., a.a.O., S. 221.

Vgl. F. Ansprenger, Politische Geschichte..., a.a.O. und J. Healy / M. Robinson, Democracy..., 14

15 Vgl. D. Kohnert, Perspektiven..., a.a.O.

Vgl. G. Lachenmann, Anmerkungen zu den gesellschaftlichen Aspekten der Strukturkrise in Afrika, DIE, Berlin 1987. 
beibehalten werden konnten. Menzel stellt sogar die These auf, daß sich "die gesamte Debatte um die Neue Weltwirtschaftsordnung und die Forderung nach vermehrter Entwicklungshilfe (...) schlichtweg als Versuch interpretieren (läßt), die Revenue der Herrschenden zu mehren und gleichzeitig von den Problemen im Lande abzulenken." ${ }^{16}$ Öffentliche Verwaltungen in Afrika südlich der Sahara stellen also keine Bürokratien im Weberschen Sinne dar. Zwar sind Staat und Verwaltung durchaus rational und funktional, aber in einem ganz anderen Sinne. Das Zielverständnis öffentlicher Verwaltungen in Afrika südlich der Sahara hat wenig mit den Verfassungsvorgaben zu tun und ist nicht mit dem von Verwaltungen in Industrieländern vergleichbar. Öffentliche Verwaltungen in vielen Ländern Afrikas südlich der Sahara sind vielmehr strukturell dysfunktional, d.h., sie lassen sich nur als Instrumente individueller Existenz- und klientelistischer Herrschaftssicherung verstehen.

\section{Maßnahmen und Initiativen zur Reform öffentlicher Verwaltungen in Afrika südlich der Sahara}

Mit den im Rahmen von Strukturanpassungsprogrammen bisher unternommenen Versuchen, die Aufgaben von Staat und Verwaltung zu begrenzen, ist es daher nicht getan. Auf diese Weise lassen sich nur die Symptome (Abwesenheit, Ineffizienz, Nepotismus, Korruption u.a.), nicht aber die Ursachen angehen, die u.a. im Fortbestehen kleptokratischer Kommandostaaten mit klientelistischen Verwaltungen, d.h. in der strukturellen Dysfunktionalität öffentlicher Verwaltungen zu sehen sind. Um diese Funktionslogik von Staat und Verwaltung in Afrika südlich der Sahara zu verändern und der "zweiten Befreiung" ${ }^{17}$ zum Durchbruch zu verhelfen, die dieses Mal - mit Wole Soyinka gesprochen - gegen den "inneren Feind" gerichtet ist, müßten die Strukturen aufgebrochen werden, mittels derer die herrschenden Eliten sich absichern und auf Kosten der Bevölkerung bereichern. Strukturanpassungsprogramme sind dabei hilfreich, wenn sie die Spielräume für Bereicherung und Willkürherrschaft begrenzen, Transparenz einführen, Verantwortlichkeiten offenlegen ${ }^{18}$ und damit den notwendigen endogenen Prozeß politischen und soziokulturellen Wandels ${ }^{19}$ unterstützen. $^{20}$

U. Menzel, Das Ende..., a.a.O., S. 53.

17

Vgl. F. Ansprenger, Afrikas zweite Befreiung: Bekenntnis zur Demokratie, in: FAZ 29.9.1993.

Dies war allerdings lange Zeit nicht der Fall. Gudrun Lachenmann kritisiert, "daß die Maßnahmen von den gesellschaftlichen Machtverhältnissen und den sozialstrukturellen Gegebenheiten sowie den Verflechtungen zwischen Staat und Gesellschaft" zu sehr abstrahierten, was dazu führe, "daß am falschen Platz gespart und damit die Effektivität der notwendigen Staatstätigkeit gemindert wird und letztendlich unerwünschte sozialstrukturelle Folgen auftreten." (G. Lachenmann, Anmerkungen..., a.a.O., S. 15.)

Kohnert vertritt die These, daß gerade die zunehmende Einschränkung der Handlungs- und Verdienstspielräume "den Brandsatz für die 'demokratische Revolution' in den afrikanischen Staaten 
Afrikanische Ansätze und Initiativen zur Verbesserung öffentlicher Verwaltungen entstanden vor allem im Kontext von Wirtschaftskrisen, Strukturanpassungspolitiken und Demokratisierungsprozessen. Einige - und nicht die schlechtesten - Reformprogramme wurden schlicht aus der Not fehlender Mittel geboren. Die Ergebnisse sind entsprechend ambivalent, aber nicht nur negativ: In Benin wurden Gesundheitszentren als "profit center" organisiert und damit überhaupt erst in die Lage versetzt, Dienstleistungen zu erbringen; in Tansania wurde das Hochschulwesen durch die Einführung kostendeckender Studiengebühren zu retten versucht; die Côte d'Ivoire hat sogar ein eigenes Kapitel zu Verwaltungsreformen in ihrem Strukturanpassungsprogramm. Die Liste könnte beliebig fortgesetzt werden. Dezentralisierung ist ein weiteres wesentliches Element von Verwaltungsreformbemühungen und in vielen Ländern Afrikas ein wichtiger Bestandteil von Reformvorhaben. Überregionale Initiativen zur Reformierung öfentlicher Verwaltungen in Afrika südlich der Sahara stellen u.a. das Observatoire des fonctions publiques africaines und das African Management Development Network dar.

Auch die Geber haben dem Selbstverständnis und dem Charakter von Staat und Verwaltung in den Ländern Afrikas südlich der Sahara erst nach langen enttäuschenden Erfahrungen mit Strukturanpassungspolitiken im Kontext der nach 1989 politischer werdenden Entwicklungszusammenarbeit Beachtung geschenkt. ${ }^{21}$ Wie die afrikanischen Regierungen hatten auch die Geber Staat und Verwaltung bis dahin als mehr oder weniger technische Problematik gesehen und entsprechend unpolitische Lösungen (Inf rastrukturmaßnahmen, Fortbildungen, Personalabbau u.a.) gesucht. Nachdem sich die Weltbank mit ihren Strukturanpassungsprogrammen und den dazugehörigen Instrumenten und Verfahren lange Zeit lediglich indirekt mit der öffentlichen Verwaltung befaßt hat, ${ }^{22}$ findet seit Ende der $80 \mathrm{er}$, Anfang der 90er Jahre mit public sector management-Maßnahmen und civil service reforms erstmals eine direkte Auseinandersetzung mit der öffentlichen Verwaltung statt. Mittlerweile sind auch hier Erfahrungen und Fortschritte gemacht worden. Der Weltbank ist zunehmend deutlich geworden, daß Reformen des öffentlichen Dienstes komplexer sein

gelegt" hat, insofern der sich herausbildende Mittelstand nicht mehr damit rechnen kann, automatisch an den Pf ründen der Staatsadministration teilzuhaben oder auch nur eine existenzsichernde Beschäftigung in diesem Sektor zu finden." (D. Kohnert, Perspektiven..., a.a.O., S. 6.)

"Wo die Verhinderung einer frühzeitigen Überzentralisierung zu einem entscheidenden Kriterium der Entwicklungsfähigkeit wird, erscheinen diese ineffizienten, hypertrophierten Entwicklungsbürokratien als ein auch sozio-kulturell bedeutsames Entwicklungshindernis." (D. Berg-Schlosser / R. Siegler, Politische Stabilität und Entwicklung - Eine vergleichende Analyse der Bestimmungsfaktoren und Interaktionsmuster in Kenia, Tansania und Uganda, Marburg 1988, S. 41.)

Vgl. P.P. Waller, Internationale Unterstützung des Reformprozesses in Entwicklungsländern im Rahmen von Auflagenpolitik und Politikdialog. Das Beispiel patrimonialer Regime in Afrika, in: H. Sautter (Hrsg.), Wirtschaftspolitische Reformen in Entwicklungsländern, Berlin 1991.

Vgl. S. Paul, Institutional Reforms in Sector Adjustment Operations, The World Bank's Experience, Washington 1990. 
müssen und längerer Zeit zur erfolgreichen Formulierung und Umsetzung bedürfen. ${ }^{23}$ Dieser Bewußtseinswandel in der Weltbank kommt auch in der stärkeren und kritischeren Auseinandersetzung mit "capacity building" und "institutional development" zum Ausdruck. Die maßgeblichen Dokumente stellen diesbezüglich die Long-Term Perspectives Study (LTPS) der Weltbank ${ }^{24}$ und die Rede des für Afrika zuständigen Vize-Präsidenten der Weltbank, Edward Jaycox, 1993 dar. ${ }^{25}$ Darüber hinaus hat die Weltbank erste Bemühungen unternommen, Verwaltungsreformen im Rahmen einer sog. "public administration adjustment operation" zu unterstützen.

Auf internationaler Ebene werden Verwaltungsreformen in Afrika südlich der Sahara darüber hinaus vor allem von der Management Development and Governance Division von UNDP, von der Global Coalition for Africa sowie von der OECD thematisiert und gefördert.

Im Rahmen der deutschen Entwicklungszusammenarbeit spielt die Verwaltungsförderung bisher erst eine geringe Rolle. Größere Beachtung findet sie erst seit der Einführung der fünf politischen Vergabekriterien 1991. Dazu zählen die Beachtung der Menschenrechte, die Beteiligung der Bevölkerung am politischen Prozeß, die Gewährleistung von Rechtssicherheit, die Schaffung einer "marktfreundlichen" Wirtschaftsordnung und die Entwicklungsorientierung staatlichen Handelns. ${ }^{26}$ Mit der Entwicklungsorientierung staatlichen Handelns ist u.a. gemeint, "in welchem Maße der Staat sich seiner Kernaufgaben annimmt und mit welcher Effizienz er sie erfüllt". ${ }^{27}$

Vgl. B. Nunberg, Public Sector Pay and Employment Reform - A Review of World Bank Experience, Washington 1989; dies., Public Sector Management Issues in Structural Adjustment Lending, Washington 1992; dies., Managing the Civil Service: What LDCs Can Learn from Developed Country Reforms, Washington 1992 sowie M. Dia, A Governance Approach to Civil Service Reform in Sub-Saharan Africa, Washington 1993.

Vgl. World Bank, Sub-Saharan Africa - From Crisis to Sustainable Growth. A Long-Term Perspective Study, Washington 1989.

Vgl. E. Jaycox, Capacity Building. The Missing Link in African Development, in: epd-Entwicklungspolitik, September 1993.

26 Vgl. C.D. Spranger, Neue politische Kriterien des Bundesministeriums für wirtschaftliche Zusammenarbeit und Entwicklung, Rede vor der Bundespressekonferenz, Bonn, 10.10.1991 und $K$. van de Sand /R. Mohs, Neue politische Kriterien des BMZ, in: E + Z, H. 10, 1991. Zur Sichtweise des BMZ bezüglich "good governance" vgl. H.P. Repnik / R. Mohs, "Good Governance", Democracy and Development Paradigms, in: Intereconomics, Jan./Feb. 1992.

Vgl. C.D. Spranger, Neue politische Kriterien..., a.a.O. 


\section{Der konzeptionelle Rahmen: Lean management}

Lean management stellt ein gegenwärtig viel diskutiertes, interessantes Reformkonzept dar, das in der japanischen Automobilindustrie entwickelt wurde, ${ }^{28}$ mittlerweile aber auch in anderen Bereichen privater und öffentlicher Produktion von Gütern und Dienstleistungen zu erheblichen Effizienz- und Qualitätssteigerungen geführt hat. Die Autoren kommen in ihrer vergleichenden Untersuchung von $90 \mathrm{Kfz}$-Produktionsstätten in 17 Ländern zu folgenden Ergebnissen: Die "schlanken" japanischen Unternehmen benötigen lediglich die Hälfte an Personaleinsatz, Investitionsaufwand für Werkzeuge und Maschinen, Produktionsfläche, Entwicklungszeit und weniger als die Hälfte an Lagerkapazität für Vor- und Zulieferprodukte als ihre europäischen und amerikanischen Konkurrenten, um mehr Produkte in größerer Vielfalt und höherer Qualität herzustellen." ${ }^{29}$ Der Erfolg dieses Managementansatzes ist nicht auf technische oder technologische Überlegenheit, geringere Personalkosten, Ausbeutung der Zulieferer oder ein spezifisches Arbeitsethos zurückzuführen, sondern auf eine intelligente Organisation der Arbeitsabläufe, eine "schlanke" Arbeits- und Produktionsweise.

Die wichtigsten Elemente des lean managements sind:

- Ausrichtung aller Unternehmensaktivitäten an der Wertschöpfungskette,

- intra- und interorganisatorische Kooperationsorientierung einschließlich der dafür erforderlichen permanenten Qualifizierung der Mitarbeiter,

- präventive und integrierte Qualitätssicherung durch Orientierung des gesamten Unternehmens auf kontinuierliche Verbesserungsaktivitäten,

- Existenz einer Unternehmenskultur, die sich durch ein beteiligungsorientiertes Management und offene Kommunikation auszeichnet.

Diese Ansätze und Instrumente sind z.T. nicht neu. Das Besondere des lean managementKonzeptes liegt in der Verbindung verschiedener, sich gegenseitig unterstützender oder sogar bedingen Faktoren der zu einem kohärenten Reformansatz.

Vgl. J.P. Womack / D.T. Jones / D. Roos, The Machine that Changed the World, New York 1990. Ebenda, S. 19.

Vgl. M.A. Fuss /L. Waverman, Costs and Productivity in Automobile Production: The Challenge of Japanese Efficiency, Cambridge/New York 1992; U. Jürgens, In Japan stößt die "Lean Production" bereits an ihre Grenzen, in: Blick durch die Wirtschaft, 19.5.1992 sowie P. Oehlke, Zur gesellschaftlichen Ambivalenz der schlanken Produktionsstrategien, in: WSI Mitteilungen, $\mathrm{H}$. 2, 1993. 


\section{Lean management als Konzept zur Reform öffentlicher Verwaltungen}

Sowohl in den Industrie- als auch in den Entwicklungsländern wird in jüngster Zeit verstärkt über die Notwendigkeit von Effizienzsteigerungen und Qualitätsverbesserungen in der öffentlichen Verwaltung diskutiert. ${ }^{31}$ Wichtige Beiträge hat dazu vor allem die sog. "new public management"-Debatte geliefert. "New public management" ist ein Obergriff für eine Reihe von Ansätzen und Tendenzen der neueren Verwaltungssteuerungs- und -reorganisationsdebatte, die weniger auf den Ressourceneinsatz als auf den Output (Qualität, Kundenzufriedenheit) öffentlicher Verwaltungen focussieren. Mit der Zielsetzung, Institutionen in die Lage zu versetzen, sich selbst zu optimieren, stehen der Prozeß der Leistungserstellung und das Umfeld von Institutionen im Mittelpunkt der Analysen und Empfehlungen von new public management-Ansätzen. Im Rahmen dieser neueren verwaltungswissenschaftlichen Debatte wird lean management auch für öffentliche Verwaltungen in Betracht gezogen.

In diesem Zusammenhang stellen sich die konstitutiven Elemente des lean managementKonzeptes wie folgt dar:

- Ausrichtung aller Tätigkeiten am Leistungserstellungsprozeß (mittels funktionaler Differenzierung von politikformulierenden und -durchführenden Aufgaben, Reorganisation des Leistungserstellungsprozesses, Definition von Aufgaben, Zielen und "Produkten", Einführung von Kosten- und Leistungsrechnung und Controlling),

- intra- und interorganisatorische Kooperationsorientierung einschließlich permanenter Qualifizierung der Mitarbeiter und Mitarbeiterinnen,

- Qualitätsmanagement und Institutionalisierung von Mitarbeiter- und Bürgerkritik,

- Entwicklung einer Verwaltungskultur, die sich durch beteiligungsorientiertes Management und offene Kommunikation auszeichnet.

Lean management zielt auch im Bereich öffentlicher Verwaltungen nicht in erster Linie auf den Abbau öffentlicher Einrichtungen, sondern vielmehr auf den an Qualitätsverbesserung ausgerichteten Umbau öffentlicher Verwaltungen. In bestimmten Bereichen (wie z.B. der Sozial-, Umwelt- und Frauenpolitik) müssen öffentliche Verwaltungen überhaupt erst

Vgl. insbes. International Labour Organization, World Labour Report 1989, Genf 1989, Kapitel 6 ("Improving public service productivity"); C. Hood, A Public Management for all Seasons?, in: Public Administration, Bd. 69, 1991; H. Hill / H. Klages (Hrsg.), Qualitäts- und erfolgsorientiertes Verwaltungsmanagement - Aktuelle Tendenzen und Entwürfe, Berlin 1993; F. Naschold/M. Pröhl (Hrsg.), Produktivität öffentlicher Dienstleistungen, Gütersloh 1994 sowie C. Pollitt / G. Bouckaert (Hrsg.), Quality Improvement in European Public Services, Concepts, Cases and Commentary, London / Thousand Oaks / New Delhi 1995. Zur neueren verwaltungswissenschaftlichen Debatte allgemein vgl. R. Koch (Hrsg.), Verwaltungsforschung in Perspektive, BadenBaden 1987. 
aufgebaut werden. Idealiter wird ein "schlanker" Staat mehr und bessere Dienstleistungen für seine "Klienten" erbringen. Daß lean management sich tatsächlich auch für öffentliche Verwaltungen eignet, belegen die positiven Erfahrungen, die unterschiedliche Verwaltungen auf kommunaler und Länderebene in Deutschland und in anderen OECD-Ländern mit dem Reformkonzept bereits gesammelt haben.

Grundsätzlich spricht also nichts gegen die Einführung von lean management in der öffentlichen Verwaltung. Im Kontext öffentlicher Verwaltung gibt es jedoch einige Spezifika, die es zu beachten bzw. zu verändern gilt. Dazu zählen die rechtlichen Rahmenbedingungen, vor allem das Dienst- und das Haushaltsrecht. Das Dienstrecht der öffentlichen Verwaltung muß so verändert werden, daß es flexible Arbeitszeiten, leistungsbezogene Bezahlung, mehrdimensionale Personalentwicklungs- und Karrieremöglichkeiten sowie zeitlich befristete Tätigkeiten im öffentlichen Dienst ermöglicht. ${ }^{32}$ Das Haushaltsrecht behindert unternehmerische Entscheidungen durch Kostenintransparenz und Inflexibilität (insbesondere unzureichende sachliche und zeitliche Übertragbarkeit von Haushaltsmitteln). Die kameralistische Buchführung sollte daher durch Budgetierungs- und Kostenrechnungsverfahren ersetzt werden, die es erlauben, Kosten auf zuzeigen und Kostenstellen (d.h. Verwaltungseinheiten) dafür zur Verantwortung zu ziehen. Anderen Besonderheiten öffentlicher Verwaltungen (wie beispielsweise das Unterworfensein unter rechtsstaatliche Bindungen und Kontrollen, die Einhaltung demokratischer Verfahren, die Berücksichtigung unterschiedlicher Interessen im Rahmen des Gemeinwohls, die Beachtung sozialstaatlicher und ökologischer Grundsätze) kann bei der Umsetzung von lean management-Reformen dadurch Rechnung getragen werden, daß sie als Ziele öffentlicher Tätigkeit definiert werden. $^{33}$

Von Effizienzsteigerungen und Qualitätsverbesserungen abgesehen, bietet die Einführung von lean management aber auch ein erhebliches Humanisierungspotential. Arbeitsplätze werden abwechslungsreicher und verantwortungsvoller, Mitarbeiter und Mitarbeiterinnen können selbstbestimmter arbeiten, werden weniger durch Vorschriften, Dienstwege und Hierarchien eingeengt, sondern aktiv und individuell gefördert. Information, Kommunikation und Austausch nehmen $\mathrm{zu}$, und damit verbessern sich auch Umgangston und "Betriebsklima". ${ }^{34}$ Befürchtungen im Zusammenhang mit der Einführung von lean

Vgl. H. Siedentopf, Der öffentliche Dienst, in: K. König, / H.J. v. Oertzen / F. Wagener (Hrsg.), Öffentliche Verwaltung in der Bundesrepublik Deutschland, Baden-Baden 1981; U. Becker / B. Krüger, Personalverwaltung und Personalführung, in: ebenda sowie $F$. Naschold, Produktivität öffentlicher Dienstleistungen, in: F. Naschold / M. Pröhl (Hrsg.), Produktivität..., a.a.O.

Vgl. H. Hill, Strategische Erfolgsfaktoren in der öffentlichen Verwaltung, in: $\mathrm{H}$. Hill / H. Klages (Hrsg.), Qualitäts- und erfolgsorientiertes Verwaltungsmanagement..., a.a.O.

34

Vgl. K.H. Große Peclum, Lean Management - primär ein Programm zur Produktivitätsförderung oder ein humanzentriertes Managementkonzept?, in: Institute for International Research (Hrsg.), Lean Management in der Verwaltung des Unternehmens, Frankfurt a.M. 1993. 
management in öffentliche Verwaltungen betreffen vor allem eine mögliche Schwächung der politischen Steuerung, unzureichende Kontrolle öffentlicher Verwaltungen und Überlastung von Arbeitnehmern. Diese Befürchtungen sind z.T. unbegründet, z.T. führen die entsprechenden Veränderungen sogar zu positiven Effekten: Verbesserung und Stärkung von Steuerung bei gleichzeitiger Begrenzung politischer Einflußnahme, Ersetzung zentraler und formaler Kontrollen durch dezentrale, ergebnisorientierte Kontrollen, qualitative Verbesserung von Arbeitsplätzen im Sinne von job enlargement und job enrichment. Lean management stellt insofern gerade auch für öffentliche Verwaltungen ein interessantes effizienz- und qualitätsverbesserndes Organisations- und Managementkonzept dar, das organisations- und kontextspezifisch umgesetzt werden sollte. Bei der Einführung kommt es in erster Linie darauf an, lernende und sich selbst optimierende Organisationen zu schaffen.

\section{Lean management als Konzept zur Reform öffentlicher Verwaltungen in Afrika südlich der Sahara}

In Entwicklungsländern Afrikas südlich der Sahara ist der Reformbedarf ungleich größer und dringender als in Industrieländern, die sich den Luxus überflüssiger Aufgabenwahrnehmung und ineffizienter Institutionen glauben leisten zu können. Der unverhältnismäßig größere Problemdruck aber stellt nicht unbedingt ein Hemmnis, sondern, wie das Beispiel Ugandas im folgenden zeigt, einen wichtigen Erfolgsfaktor dar, der die effektive Umsetzung von umfassenden Reformen begünstigt.

Das lean management-Konzept scheint gerade für Entwicklungsländer besonders geeignet, da es wesentliche Defizite der öffentlichen Verwaltungen abzubauen vermag ${ }^{35}$ und nicht mehr Institutionen, mehr Reglementierung und mehr Mittel erfordert, sondern - ausgehend von dem, was vorhanden ist - darauf zielt, eine sich selbst tragende Verbesserungsdynamik in Gang zu setzen ("encourage organizations to help themselves" ${ }^{36}$ ). Im Unterschied zu vielen anderen Verwaltungsförderungskonzepten stellt es nicht auf imposante Infrastrukturen, komplexe Regulierungen und entsprechend umfassenden Know-how- und Mitteltransfer ab, sondern auf eine sich weitgehend selbsttragende und selbstoptimierende Arbeitsorganisation. Größere Eigenverantwortlichkeit einzelner Verwaltungseinheiten stärkt diese gegen zentralstaatliche Instrumentalisierung und trägt damit auch zu einem sukzessiven Abbau struktureller Dysfunktionalität bei.

Vgl. A. Stockmayer, Staatliche Verwaltung..., a.a.O. und G. Mutahaba / R. Baguma / M. Halfani, Vitalizing African Public Administration..., a.a.O.

36

M. Moore, Promoting Good Governance by Supporting Institutional Development, in: IDS Bulletin, H. 2, 1995, S. 94. 
Gleichzeitig ist die Gefahr, daß öffentliche Verwaltungen übermäßig abgebaut und Versorgungs- und Dienstleistungslücken entstehen könnten, in Entwicklungsländern Afrikas südlich der Sahara wesentlich geringer als in Industrieländern, da Staat und öffentliche Verwaltung in den meisten entwicklungspolitisch wichtigen Bereichen, wie z.B. im Umwelt-, Bildungs-, Gesundheits- und Sozialbereich, ohnehin kaum präsent sind. Lean management-Reformen werden für die Bevölkerungsmehrheit in Entwicklungsländern vor allem positive Auswirkungen haben, indem sie dazu beitragen,

- die zur Verfügung stehenden knappen Ressourcen auf die wichtigsten Tätigkeiten zu konzentrieren,

- Kommandostrukturen durch dezentrale Eigenverantwortlichkeiten zu ersetzen,

- Rechenschaftspflicht von Regierungen und Verwaltungen einzuführen,

- Spielräume für Bereicherung, Klientelismus und Korruption zu reduzieren,

- Transparenz von Staats- und Verwaltungshandeln zu verbessern und eine

- größere Einflußnahme der Bevölkerung zu ermöglichen.

Gleichwohl darf nicht übersehen werden, daß in zahlreichen personalstarken Verwaltungsbereichen vieler Entwicklungsländer erheblicher Personalabbau stattgefunden hat und weiter stattfinden wird. Dies geschieht nicht nur auf Kosten der aktuellen Stelleninhaber, sondern vor allem auf Kosten der jüngeren Leute, die kaum noch Aussicht auf eine Beschäftigung im öffentlichen Dienst haben. Ein Großteil dieser oft brachial und ohne jeglichen Reformansatz durchgeführten Personalabbaumaßnahmen aber hat wenig mit lean management-Reformen zu tun und daher auch nur bedingt zu effektiven, geschweige denn qualitativen Verbesserungen der öffentlichen Verwaltungen geführt. Entsprechende Maßnahmen implizieren nicht nur erhebliche soziale Kosten, sondern sind auch politisch problematisch, da sie die Akzeptanz gerade demokratisch legitimierter Regierungen, die die Last unvertretbarer Politiken der Vergangenheit abzutragen haben, zu unterminieren drohen. In diesen Fällen ist vor allem auf die oftmals zu beobachtende komplementäre Enstehung und Entwicklung zivilgesellschaftlicher Organisationen und Strukturen zu hoffen, die neue Arbeitsplätze schaffen, Dienstleistungslücken füllen und eine politische watch-dogRolle übernehmen.

\section{Länderfallstudien Benin und Uganda}

\section{Verwaltungsreformen in Benin}

Benin ist eines der wenigen Länder in Afrika südlich der Sahara, das sich 1990/91 auf friedliche Weise von einer Diktatur zu einer Demokratie gewandelt hat. Seit nunmehr sechs Jahren wird um die Einführung und Konsolidierung demokratischer Institutionen und 
Verfahren gerungen. Abgesehen davon, daß demokratische Institutionen und Verfahren eingeführt und 1995 Parlamentswahlen durchgeführt wurden, sind die Ergebnisse bisher allerdings wenig beeindruckend. An den Dominanz- und Ausbeutungsstrukturen (Staatsklasse versus Bevölkerungsmehrheit) hat sich wenig geändert, ein gesellschaftlicher Umbau hat nicht stattgefunden. Die Teilhabe eines Großteils der Bevölkerung an Politik, Wirtschaft und Verwaltung wurde trotz formaler Demokratisierung erfolgreich unterbunden. Die Staatsklasse hat sich lediglich darauf verlegt, ihr Erscheinungsbild zu verändern, d.h., sie gibt sich demokratisch, behindert aber gleichzeitig effektive Demokratisierung, wo immer sie kann. Willkür, Korruption und Vetternwirtschaft haben nach Meinung des Beniner Bischofs da Souza, der gleichzeitig Präsident des Hohen Rates, des höchsten Verfassungsorgans Benins, ist, sogar eher zu- als abgenommen. ${ }^{37}$ Der gesellschaftliche Reformprozeß befindet sich mittlerweile in einer Phase der Stagnation.

Die Hauptprobleme der öffentlichen Verwaltung sind strukturelle Dysfunktionalität, die in Klientelismus und Nepotismus zum Ausdruck kommt, Korruption, die auf die ungenügende Bezahlung im öffentlichen Dienst zurückzuführen ist, und die Reformunwilligkeit der Beniner Staatsklasse, die an Machterhaltung, nicht aber an Reformen interessiert ist, und daher auch an zentralistischen Strukturen festhält. Staat und Verwaltung treten in Benin als Kommandostruktur und Ausbeutungsapparat in Erscheinung. Es ist insofern verständlich, daß sich die Mehrheit der Bevölkerung dem staatlichen Zugriff durch die Verwaltung soweit wie möglich zu entziehen versucht ${ }^{38}$ und Reformvorhaben, die in der Vergangenheit oft dazu dienten, subtilere Unterdrückungs- und Ausbeutungsmechanismen einzuführen, skeptisch gegenübersteht.

Die öf fentliche Verwaltung ist aber nicht nur ein Problem für sich, sondern blockiert auch politische und wirtschaftliche Entwicklungen in vielen anderen Bereichen. Die Reformierung der öffentlichen Verwaltung ist daher kein Selbstzweck, sondern notwendige Voraussetzung für erfolgreiche Reformmaßnahmen in anderen Sektoren.

Die Beniner Verwaltungsreformvorhaben zielen auf eine Optimierung der öffentlichen Verwaltung - in Beniner Texten ist sogar teilweise explizit von Verschlankung ("allège$m e n t ")$ die Rede. Sie umfassen die drei folgenden Reformbereiche:

- Abbau des öffentlichen Dienstes und Reorganisation von Ministerien sowie

- Dezentralisierung, die mit einer Reform der Territorialverwaltung verbunden wird.

- Die Auslagerung bisher ministerieller Aufgaben in parastaatliche Strukturen läßt sich als dritter Reformbereich definieren, auch wenn die entsprechenden Maßnahmen unabhängig von der Verwaltungsreform konzipiert und durchgeführt werden.

37 38

Vgl. D. Kohnert, Perspektiven..., a.a.O.

Vgl. ebenda. 
Wichtigstes Ergebnis der Analyse der Beniner Reformpolitik ist, daß die (alte und neue) herrschende politische Klasse kein Interesse an einer Reformierung des Staatsapparates hat und Verwaltungsreformen daher nur soweit vorangetrieben und umgesetzt werden können, wie die Geber auf Reformen bestehen und diese unterstützen. Vor diesem Hintergrund wird verständlich, warum die Reformmaßnahmen die grundlegenden Strukturdefizite außer acht lassen, unzureichend miteinander verbunden, schlecht sequenziert und im Ergebnis wenig erfolgreich sind. ${ }^{39}$

Zwar gibt es mit der offenen Informations- und Zielvereinbarungspolitik zu Beginn des Demokratisierungsprozesses (1989/90) und dem Dezentralisierungsvorhaben einige interessante Elemente, die als "lean" bezeichnet werden könnten, doch entspricht der Großteil der Reformelemente und vor allem der Reformprozeß nicht den Kriterien von lean management. Als Mißerfolgsfaktoren, die zum Scheitern der Verwaltungsreformen beigetragen haben, lassen sich die folgenden nennen:

- Fokussierung auf marginale statt auf zentrale Probleme,

- unzureichendes Management der Reformen,

- mangelnde Verknüpfung und falsche Sequenzierung der Reformmaßnahmen,

- fehlende Reformbereitschaft sowie

- problematische Trägerstrukturen.

Da die Reformbereitschaft vieler Akteure bereits verspielt und die Hoffnung von Teilen der Bevölkerung enttäuscht wurde, ${ }^{40}$ sind die Erfolgsaussichten zukünftiger Reformvorhaben nachhaltig beeinträchtigt.

Trotz des weitgehenden Scheiterns der Verwaltungsreformvorhaben in Benin lassen sich Erfolgsfaktoren benennen, die dazu beigetragen haben, daß Verwaltungsreformen überhaupt auf die politische Agenda gekommen sind:

- Offenheit von Information, politischer Mitbestimmung und Zielvereinbarung, die zu Anfang des Beniner Reformprozesses vorherrschte und sich vor allem in der Nationalkonferenz, den Generalständen ("Etats généraux") und der neuen Verfassung konkretisierte,

- Ansätze eines politischen Differenzierungsprozesses (Herausbildung unterschiedlicher Fraktionen),

- die anfangs kritische Begleitung und Unterstützung des Reformprozesses durch die Geber.

Vgl. dazu ausführlich $H$. Lingnau, Lean management als Konzept zur Reform öffentlicher Verwaltungen in Afrika südlich der Sahara - Schlußfolgerungen aus den Verwaltungsreformen Benins und Ugandas, Köln 1996, Kap. 5.

40

Vgl. E. Ahohe /J. Azondekon / C. Konnigui /L. Attolou /V. Zocli, Les expériences de réforme de la fonction publique menées au Bénin depuis 1986, Cotonou 1995. 
Ohne die Notwendigkeit der Unterstützung der Verwaltungsreformen durch die Geber in Frage stellen zu wollen, ist die Rolle der Geber im Kontext der Beniner Reformpolitik vor allem in zweierlei Hinsicht problematisch: Zum einen dominieren die Geber den Reformprozeß, zum anderen sind sie nicht in der Lage, die Beniner Regierung und Staatsklasse zu effektiver Reformpolitik zu bewegen. Die Dominanz der Geber ist sowohl im konzeptionellen Bereich als auch bei der Implementierung festzustellen. ${ }^{41}$ Laxheit bei der Einforderung effektiver Reformschritte ist vor allem der Weltbank vorzuwerfen, die ungeachtet heftiger Kritik anderer Geber Darlehen ausgezahlt hat, obwohl allgemein bekannt war, daß die damit verbundenen Auflagen nicht erfüllt waren. Diese Haltung erklärt sich aus dem Mittelabflußdruck, dem die Geber insbesondere gegenüber vermeintlich reformorientierten Entwicklungsländern ausgesetzt sind. Auf die Reformpolitik hat das fatale Auswirkungen: Indem die Beniner Verantwortlichen erleben, daß Unterstützung auch dann gewährt wird, wenn die allgemein als entwicklungsfeindlich und reformbedürftig erkannten Strukturen unverändert weiterbestehen, tendieren die Chancen einer effektiven Umsetzung von Reformmaßnahmen gegen Null. Der damit verbundene Verlust von Glaubwürdigkeit wird nur schwer wettzumachen sein und auch zukünftige Reformvorhaben beeinträchtigen. In Zukunft kommt es darauf an, eindeutige Ziel- und Zeitvorgaben zu vereinbaren und auf deren Einhaltung auch dann zu bestehen, wenn die Beniner Regierung versucht, die Geber zum Sündenbock zu stempeln. Gleichzeitig sollten sich die Geber aus der Feinsteuerung heraushalten und damit allen Beteiligten deutlich machen, wer für welche Maßnahmen die Verantwortung trägt.

\section{Verwaltungsreformen in Uganda}

Uganda ist ein Beispiel dafür, daß es auch in Afrika südlich der Sahara Regierungen mit ernsthaften und erfolgreichen Reformbemühungen gibt. Das Land fand bis vor kurzem wenig Beachtung, da die sozialistisch inspirierte Regierung aus einer Guerilla-Bewegung hervorgegangen war. Seit der Regierungsübernahme 1986 betreibt die Regierung unter Präsident Yoweri Museveni eine wirkungsvolle Reformpolitik, die zunehmend auch von westlichen Gebern als Erfolgsfall angesehen wird.

Die Ergebnisse dieser Reformpolitik sind beeindruckend: Bürgerkrieg, Terror und Folter sind weitgehend beendet, ${ }^{42}$ Staat, Verwaltung und Wirtschaft werden liberalisiert, und die Wachstumsraten des BSP liegen seit der Regierungsübernahme bei 5-8 \%. Uganda gilt neben Ghana als Musterland der Strukturanpassung. ${ }^{43}$ Auch politisch findet ein eindrucks-

\footnotetext{
41 Vgl. ebenda.

42 Vgl. u.a. E.A. Brett, Providing for the Rural Poor..., a.a.O.

43 Vgl. M. Obera / A. Rake, Spotlight Uganda - Museveni's New Deal, in: New African, Dezember 1993.
} 
voller Prozeß politischer Öffnung und Demokratisierung statt. 1994 haben die ersten landesweiten freien und geheimen Wahlen in der Geschichte des unabhängigen Uganda stattgefunden, 1996 sind Parlaments- und Präsidentschaftswahlen gefolgt. Der Erfolg des von der ugandischen Regierung eingeschlagenen Weges wird von allen seriösen Beobachtern bestätigt.

Ein wesentlicher Aspekt der Reformpolitik ist der Umbau des Staatsapparates und seiner Funktionen. Staat und Verwaltung waren bei Übernahme der Regierung durch das National Resistance Movement (NRM) 1986 in desolatem Zustand und weitgehend kompromit tiert. $^{44}$ Das distanzierte bis feindliche Verhältnis, das die Bevölkerung schon vor der Kolonialzeit zu Staat und Verwaltung hatte, ${ }^{45}$ wandelte sich während der Kolonialzeit und insbesondere während der Terrorregime von Idi Amin und Milton Obote, unter deren die Bevölkerung willkürlicher Folter, Verfolgung, Vertreibung sowie Raub und Mord von seiten des Staates ausgesetzt war, in tiefes Mißtrauen. ${ }^{46}$ Die Bevölkerung lastet die Verbrechen vor allem den Politikern an, ${ }^{47}$ aber auch den Verwaltungsbeamten, die den Terrorregimen treu dienten. ${ }^{48}$

Das Vertrauen der Bevölkerung in Politik und Verwaltung läßt sich nur langsam wiederaufbauen. Wichtigstes Element dieses Prozesses ist die politische Partizipation im Rahmen der von der Bevölkerung direkt gewählten Resistance Councils, die die staatliche Verwaltung kontrollieren und die Bevölkerung vor Machtmißbrauch durch Staatsbeamte schützen sollen.

Da gewalttätige Übergriffe des Staates auf die Bevölkerung seit 1986 unterbleiben, sind die gravierendsten Probleme der öf fentlichen Verwaltung Ugandas strukturelle Dysfunktionalität und Korruption. ${ }^{49}$ Sie sind vor allem auf eine pervertiertes Selbstverständnis, entsprechend absurde Strukturen und viel zu geringe Bezahlung der im öffentlichen Dienst Beschäftigten zurückzuführen. "The problem was not outmoded regulations, but that existing regulations were systematically abused by managers and staff alike. Government became little more than a criminal conspiracy against the public." 50

44

45

46

47

48

49

50

Vgl. International Monetary' Fund, Uganda: Adjustment with Growth, 1987-94, Washington 1995.

Vgl. S.R. Karugire, A Political History of Uganda, Nairobi, London 1980.

Vgl. E.A. Brett, Providing for the Rural Poor..., a.a.O.

Vgl. Transparency International, Report of the Mission of TI to Uganda, Berlin Dezember 1994.

Vgl. I. Kabumba, The Contribution of Managers to the Survival of Dictatorship in Uganda, Kampala 1992.

Vgl. T. Banyenzaki, Time to Reform Public Service, in: The New Vision, 19.5.1995.

Ministry of Public Service, Management of Change - Context, Vision, Objectives, Strategy and Plan, Kampala 1994, S. 5. 
Mit den wichtigsten Verwaltungsreformvorhaben - der Restrukturierung der öffentlichen Verwaltung verbunden mit einem Abbau des öffentlichen Dienstes, der Dezentralisierung und verschiedenen qualitätsverbessernden Maßnahmen ${ }^{51}$ - geht die ugandische Regierung die wesentlichen Defizite an und legt die Kontrolle des Staatsapparates in die Hände der Bürger, die als Konsumenten und Wähler das umfassendste Monitoring- und Kontrollsystem darstellen, das überhaupt vorstellbar ist. ${ }^{52}$ Dank dieser Politik scheint es gelungen zu sein, die Kluft zwischen der herrschenden Elite und dem Großteil der Bevölkerung erstmalig zu überbrücken: "Ebyaffe", d.h. "unsere Sachen" ist zum vorherrschenden Begriff der politischen Diskussion und damit auch einer neuen politischen Einstellung in Uganda geworden. ${ }^{53}$

Das "management of change" ${ }^{54}$ der ugandischen Regierung zur Konzipierung und Umsetzung der Reformpolitik ist für alle Beobachter beeindruckend. Dies gilt auch und besonders für den Bereich der öffentlichen Verwaltung, in dem die ugandische Regierung erstaunlich kreativ und dezidiert Reformen konzipiert und vorantreibt. Das heißt nicht, daß es nicht auch Unzufriedenheit und Kritik (vor allem von seiten der Entlassenen und De-Privilegierten) gibt, auf die die Regierung gewisse Rücksichten zu nehmen hat. Zu den "Verlierern" zählen v.a. die "alten Eliten, die ihre lukrativen Posten bei Staatsbetrieben, Banken oder marketing boards zur persönlichen Bereicherung ausgenutzt bzw. die von der Enteignung indischer Unternehmen und Immobilien profitiert hatten. Sie gehören zu den heftigsten Kritikern des Reformkurses, zumal sich die Regierung beharrlich weigert, ihnen Kredite zur Verfügung zu stellen, mit denen sie sich an der Privatisierung der Staatsbetriebe beteiligen könnten. In ihrer Kritik, die sie pikanterweise mit solchen Schlagworten wie 'imperialistische Überfremdung der Wirtschaft' oder 'Ausverkauf des Volkseigentums' begründen, erhalten sie dabei nicht selten Schützenhilfe von solchen altlinken Intellektuellen, die nach wie vor einer staatlichen Planwirtschaft das Wort reden. Auf eine Resonanz bei der Bevölkerung stoßen die populistischen Parolen der 'mafuta mingi' allerdings kaum. Die meisten Ugander wissen nur zu gut, daß sie von ihrem 'Volkseigentum' nie etwas hatten, es sei denn das zweifelhafte Privileg, mit ihren Steuergeldern für dessen Verluste aufkommen zu dürfen." ${ }^{55} \mathrm{Daß}$ sie die Reformmaßnahmen auch vor den Wahlen zur Verfassunggebenden Versammlung (1994) und vor den Parlaments- und Präsidentschaftwahlen (1996) nicht ausgedünnt oder ausgesetzt hat, verdeutlicht, daß es sich um ernstgemeinte Reformvorha-

51 52

So Transparency International, die vom "most powerful monitoring system available" sprechen "that of consumers and the voters". (Transparency International, Report ..., a.a.O., S. 17.)

53

54

55

F. Ballot, Ökonomisches Musterland Afrikas? Die Wirtschaftspolitik der Regierung Museveni, in: blätter desiz3w, Nr. 169, 1994. 
ben einer wirklich reformorientierten Regierung handelt. Auch Liden stellt in diesem Zusammenhang fest: "No one, save some representatives of Milton Obote's old party, Uganda People's Congress, would doubt General Museveni's sincerity in his search of a system that gives ordinary people the power to elect and dismiss governments through their votes." ${ }^{56}$

Beeindruckend ist ferner, daß die ugandische Regierung die öffentliche Verwaltung nicht nur reduziert und restrukturiert, sondern mit unterschiedlichen Maßnahmen zur Qualitätsverbesserung (codes of conduct, results-oriented management, Inspectorate General of Government) Vorsorge getroffen hat, um diese Erfolge abzusichern und zu perfektionieren. Die verschiedenen Vorhaben zur Reform der öffentlichen Verwaltung weisen viele lean management-Elemente auf, die von den beteiligten Akteuren viel selbstverständlicher als in Deutschland diskutiert und angegangen werden. Daß noch viel zu tun bleibt und der Reformprozeß noch einige Zeit braucht, um wirklich greifen, d.h. zu einer veränderten Verwaltungskultur führen zu können, ist allen Beteiligten klar. Die lean managementKonzepte und Instrumente werden dabei aber nicht (wie in einigen Industrieländern) als abwegig oder utopisch angesehen, sondern als hilfreich und zwingend erforderlich.

Die größten Schwierigkeiten, die im Rahmen der Verwaltungsreform entstanden sind, resultieren aus einer unzureichenden (inhaltlichen und zeitlichen) Verknüpfung der verschiedenen Vorhaben. Diese piecemeal-policy ist in nicht unerheblichem Maße auf die disparaten Einzelinteressen und entsprechende Politiken der Geber zurückzuführen und kann von der ugandischen Regierung, deren Budget zu über 50 \% von Außenfinanzierung abhängig ist, nur sehr bedingt ausgeglichen werden.

Die wichtigsten Erfolgsfaktoren im Falle Ugandas sind

- die Reformorientierung des Präsidenten, der die Ursachen für die Probleme des Landes nicht bei anderen sucht, sondern endogene Faktoren verantwortlich macht, sich der "good governance" verschrieben hat, einen neuen Politikstil und Moral in das öffentliche Leben eingeführt hat und selbst vorlebt und dies auch von allen anderen Personen des öffentlichen Lebens verlangt,

- die Reformorientierung wichtiger Mitglieder der Regierung und der Ministerien,

- die externe Unterstützung der Reformpolitik, die großzügig die notwendigen Ressourcen zur Verfügung gestellt, aber auch die Durchführung der Maßnahmen kritisch begleitet und damit der Regierung Argumente und Rückhalt für eine stringente Umsetzung auch unpopulärer Maßnahmen geboten hat,

- die Existenz des Resistance Council-Systems, das der Bevölkerung Partizipation ermöglicht und somit nicht nur half, den Wiederaufbau zu organisieren, sondern auch

56

J. Liden, On Road to Democracy, but Obstacles Remain, in: International Herald Tribune, 28.9.1994. 
und vor allem Vertrauen in Staat und Verwaltung wiederzugewinnen und demokratische Verfahren einzuüben,

- die richtige Sequenzierung der Einzelmaßnahmen,

- die Komplementarität und parallele Durchführung der verschiedenen Reformmaßnahmen,

- die Informationspolitik, die eine offene Diskussion der Vorhaben mit den betroffenen Akteuren und darüber hinaus auch in der Öffentlichkeit und mit den Gebern ermöglicht hat,

- letztlich wohl aber auch das unendliche Leid, das durch die jahrzehntelangen Terrorherrschaften verursacht wurde ("Traumatisierung") und das bei weiten Teilen der Bevölkerung zur Einsicht geführt hat, daß sich Strukturen und Verfahren politischer Auseinandersetzung verändern müssen.

Statt piecemeal-Maßnahmen einzelner Geber in einzelnen Bereichen bedarf es umfassender, vertrauensvoller Unterstützung in Form von Schuldenerla ${ }^{57}{ }^{57}$ vermehrter nicht-projektgebundener Finanzierungen sowie der Bereitschaft zu unkonventionellen Maßnahmen, wie z.B. der Finanzierung von Abfindungen und Gehaltserhöhungen. Es muß festgestellt werden, daß Uganda zur Zeit mit dem Management der vielen Einzelprojekte überfordert ist: Die Weltbank konstatiert "poor coordination of donor activities by the Government of Uganda". Dies ist u.a. darauf zurückzuführen, daß die Geber in erster Linie ihre partikularen Interessen vertreten, die Konzipierung und Umsetzung ihrer Projekte dominieren und einen "let's get on with the job"-Ansatz praktizieren, der die Prioritäten, Verfahren, Zuständigkeiten und Engpässe der ugandischen Seite ignoriert. ${ }^{58}$ Die Notwendigkeit solcher Maßnahmen wird von Transparency International, Regierungsvertretern, Gebern und der Weltbank gesehen: "It is the view of government officials, donor agencies and project managers that the top-ups are essential to keep the civil servants on the job." ${ }^{59}$ DANIDA zahlt den "heads of department" im Rakai District entsprechende Zulagen im Rahmen ihrer Unterstützung der Dezentralisierung. ${ }^{60}$ Das bedeutet nicht, daß die Geber ihre bisherige "supervisory role" aufgeben sollten, die sich in Ermangelung funktionierender interner Kontrollmechanismen als wichtig herausgestellt hat. "External checks and balances imposed upon the state by autonomous groups in the economy and civil society are essential if those in control are not to succumb to the temptations of office and use their power to

Trotz der großzügigen Bereitstellung neuer EZ-Mittel trägt Uganda an der aus den Amin- und Obote-Zeiten ererbten Schuldenlast so sehr, daß mit einem negativen Ressourcentransfer gegenüber dem IWF gerechnet wird. Vgl. Oxfam, Multilaterale Schulden als Entwicklungsblockade Das Verschuldungsprofil Ugandas, in: blätter des iz3w, Mai 1994.

59 Vgl. Transparency International, Report..., a.a.O. S. 21.

60

Vgl. The New Vision, 25.5.1995. 
advance their own rather than the nation's interests. In Uganda, foreign donors have played a dominant supervisory role, while indigenous representative structures (...) have as yet lacked the resources and the status to exert a powerful influence." 61

\section{Schlußf olgerungen}

Die Länderfallstudien zeigen, daß Verwaltungsreformen nicht nur notwendig, sondern in der Tat auch realisierbar sind. Sie sind vor allem dann erfolgreich, wenn sie mit umfassenden Maßnahmen auf die Überwindung struktureller Dysfunktionalität zielen und von reformorientierten Regierungen vorangetrieben werden. "Political commitment" wird zunehmend als "key cause (...) of organizational performance in developing countries" angesehen. ${ }^{62}$ Das Beispiel Ugandas zeigt, wie phantasievolle Verwaltungsreformen effektiv umgesetzt werden können. Gelingt es jedoch nicht, die Herrschaft sich selbst bereichernder Staatsklassen aufzubrechen und deren Macht zu begrenzen, lassen spürbare Verwaltungsreformen und effektive Demokratisierung wie im Fall Benins weiter auf sich warten.

Aus den beiden Länderfallstudien läßt sich schlußfolgern, daß für den Erfolg von Verwaltungsreformen neben Reformorientierung und Reformwille auch die richtige Ausrichtung von Reformpolitiken (auf strukturelle Dysfunktionalitäten und grundlegende Defizite) sowie die Konsistenz und die Kohärenz der Reformpakete und -maßnahmen wichtig sind. Das lean management-Reformkonzept ist für Verwaltungsreformen in Afrika südlich der Sahara keineswegs abwegig. Im Gegenteil, erfolgreich oder erfolgversprechend sind in Benin und Uganda insbesondere diejenigen Maßnahmen, die lean management-Elemente umfassen oder sogar explizit als solche bezeichnet werden. Angesichts dieses Befundes läßt sich die These vertreten, daß die new public management-Debatte und insbesondere das Instrumenten-Set "lean management" für zukünftige Verwaltungsreformvorhaben - und zwar sowohl für afrikanische Regierungen als auch für Institutionen der Entwicklungszusammenarbeit - Orientierungshilfe und Anleitung sein können.

Unmittelbare Erfolge können allerdings nur in wenigen Bereichen erwartet werden. Erst einmal führt die Reformierung öffentlicher Verwaltungen, die Machtzentrum und Herrschaftsapparat von autoritären Regierungen darstellen, zu erheblichen Turbulenzen. Es dauert vermutlich Jahre, wenn nicht Jahrzehnte oder Generationen, bis ein grundlegender Reformprozeß als gesichert gelten kann. Bis dahin sind Reformprozesse und Ergebnisse fragil.

61

E.A. Brett, Rebuilding Organisation Capacity in Uganda under the National Resistance Movement, in: The Journal of Modern African Studies, Bd. 32, 1994, H. 1, S. 55.

62

Vgl. M. Moore, Promoting Good Governance..., a.a.O., S. 95 und die dort angegebene Literatur. 
Sowohl in Uganda als auch in Benin haben die Reformmaßnahmen trotz aller Schwierigkeiten und Turbulenzen und auch ungeachtet der z.T. vielfältigen Defizite dazu beigetragen, daß

- Aufgaben und Legitimation öffentlicher Verwaltungen überhaupt diskutiert werden,

- Unterdrückung und Ausbeutung durch öffentliche Verwaltung (ansatzweise) diskreditiert wurden,

- effektivere und bessere Leistungserbringung im gesamtgesellschaftlichen Interesse verstärkt eingefordert und durch verbesserte Rechenschaftspflicht und Kontrollen, Schaffung von Anreizstrukturen und Wettbewerbssituationen auch faktisch befördert werden.

Darüber hinaus sind die Erfolgsaussichten nur länder- oder sogar reformspezifisch einschätzbar. Der Reformdruck allerdings ist so groß und zwingend, daß Verwaltungsreformvorhaben in den meisten Ländern Afrikas südlich der Sahara wohl kaum vermieden werden können. Die bisherigen Strukturen, Politiken und Ausweichmanöver sind nicht mehr durchzuhalten. Viele Staaten sind schlichtweg bankrott. Der politische Wandel und die Durchführung von Reformvorhaben in Benin wurden beispielsweise dadurch ausgelöst, daß der Staat seinen Beamten und Angestellten keine Gehälter mehr auszahlen konnte. In den Ländern, die mit den Bretton-Woods-Institutionen kooperieren, sehen die Strukturanpassungsprogramme Restrukturierungen und deutliche Reduzierungen des Verwaltungsapparates vor. In den übrigen Ländern dürfte dann, wenn die Außenfinanzierung im Zuge der politischer werdenden Entwicklungszusammenarbeit schwieriger wird, auch der Druck wachsen, öffentliche Verwaltungen zu reformieren.

Von außen induzierter Reformdruck kann aber nur Auslöser oder Katalysator von Reformprozessen sein. Damit Reformen erfolgreich sein können, bedarf es weiterer Erfolgsvoraussetzun-gen, zu denen vor dem Hintergrund der Fallstudien Benin und Uganda vor allem die folgenden zählen:

- die Beachtung von entwicklungsländerspezifischen Kontextbedingungen (Existenz massiver Strukturdefizite, Vorherrschen eines anderen Selbstverständnisses, größere Reformresistenz etc.),

- die Berücksichtigung der Handlungsrationalitäten und Interessen der Akteure (Reformorientierung der Akteure und der Staatsklasse, die in eigenen Reforminitiativen zum Ausdruck kommt und deren Umsetzung von ausreichend starken gesellschaftlichen Reformkräften eingefordert wird), ${ }^{63}$

"For the realization of democracy in any country, whether in Africa or elsewhere, cannot be without the simultaneous constitution of a democratic subject." (M. Mamdani, Conceptualising State and Civil Society Relations. Towards a Methodological Critique of Contemporary Africanism, in: C. Auroi (Hrsg.), The Role of the State in Development Processes, London 1992, S. 22.) 
- die Ausrichtung von Reform- und Unterstützungsmaßnahmen an einer veränderten Verwaltungskultur, die zu laufender Selbstverbesserung beiträgt, statt an Kleinstprojekten,

- die Gewährung umfassender Unterstützung (Sektor- und Programm- statt Projekthilfe, Bereitschaft zur zeitweiligen Finanzierung laufender Kosten wie Abfindungen, Lohnkosten, Gehaltserhöhungen).

\section{Weitere Erfolgsfaktoren sind}

- die Anerkennung der Existenz unterschiedlicher Interessen und des Prinzips eines gewaltfreien Interessenausgleichs durch die beteiligten Akteure,

- die hochrangige politische Unterstützung der Verwaltungsreformen,

- die Beteiligung aller Betroffenen,

- die Einbeziehung der Öffentlichkeit,

- die Festlegung einer eindeutigen Umsetzungsverantwortung,

- die Vereinbarung von Zielen,

- die Sicherstellung von Mindesteinkommen im öffentlichen Dienst sowie die Gewährung zusätzlicher (Leistungs-)Anreize,

- die "politische" Konditionierung von EZ-Leistungen über selbstverständliche Verwendungs- und Erfolgskontrollen hinaus, zumindest solange, wie es keine effektiven internen Kontroll- und Korrekturmechanismen gibt.

\section{Empfehlungen für die Entwicklungszusammenarbeit}

Als externer und (idealiter) interessenloser Akteur qualifiziert sich die Entwicklungszusammenarbeit bei der Unterstützung von Verwaltungsreformen besonders für eine Moderatorenrolle. Diese sollte sie bereits in der Phase der Problemsuche und Problemdefinition wahrnehmen. Bei den unvermeidlichen Auseinandersetzungen im Zuge der Umsetzung sollten externe Moderatoren insbesondere auf eine Versachlichung von Debatten drängen und zur Entpolitisierung von Auseinandersetzungen beitragen. Alle Erfahrungen mit Unternehmens- und Verwaltungsreformen belegen, daß die Einbeziehung Externer als sachverständige Moderatoren von großer Bedeutung für den Erfolg von Reformvorhaben ist.

Der Transfer positiven "Wissens" und deutscher Modelle sowie klassische Aus- und Fortbildungsmaßnahmen hingegen sind wenig hilfreich, u.U. sogar kontraproduktiv und sollten nur dann vorgesehen werden, wenn wirklicher Bedarf im Rahmen einer umfassenden 
Unterstützung identifiziert wird. In den meisten Fällen dürften die Engpässe und der Unterstützungsbedarf woanders liegen.

\section{Technische Zusammenarbeit}

Im Rahmen der Technischen Zusammenarbeit (TZ) könnte Unterstützung beispielsweise mit folgenden Instrumenten angeboten werden: Produktion von Ideen, Ausarbeitung angepaßter Konzepte, Management von Veränderungs- und Restrukturierungsprozessen, Moderation von Gesprächen, back-stopping.

Die inhaltliche Ausrichtung von Verwaltungsfördermaßnahmen sollte sich am Bedarf des betreffenden Landes orientieren. Dennoch lassen sich vor dem Hintergrund des lean management-Konzeptes Themenbereiche nennen, die besonders wichtig sind. Dazu zählen

- die Verkleinerung und Restrukturierung ("streamlining") von öffentlichen Institutionen im Sinne des lean management (Ausrichtung aller Tätigkeiten und Strukturen am Leistungserstellungsprozeß) mittels

- funktionaler Differenzierung von politikformulierenden und -durchführenden Aufgaben,

- Definition von Aufgaben, Zielen und "Produkten",

- entsprechender Reorganisation von Leistungserstellungsprozessen,

- Einführung von Kosten- und Leistungsrechnung sowie von Controlling,

- die Verbesserung der intra- und interorganisatorischen Kooperationsorientierung sowie die Qualifizierung von Mitarbeitern des öffentlichen Dienstes,

- die Einführung von Qualitätsmanagement und die Institutionalisierung von Mitarbeiterund Bürgerkritik sowie

- die Entwicklung einer beteiligungsorientierten Verwaltungskultur.

Darüber hinaus gibt es Bereiche, in denen die deutsche EZ über komparative Vorteile oder besondere Handicaps verfügt. Komparative Vorteile bestehen zweifelsohne im Schlüsselbereich Dezentralisierung, in dem die Bundesrepublik über viel Erfahrungswissen verfügt. Auch zur Stärkung des Wettbewerbsgedankens in der öffentlichen Verwaltung könnte die deutsche EZ dank der Erfahrungen beitragen, die in der Bundesrepublik mit der Durchführung von Wettbewerben bereits gemacht wurden. Aus den Bereichen Steuer- und Haushaltswesen sollte sich die deutsche Verwaltungsförderung im Unterschied zu den o.g. Themenkreisen heraushalten, da die deutsche Steuerverwaltung keineswegs vorbildlich ist. Auch die in Deutschland praktizierte Kameralistik ist nicht nachahmenswert, sondern behindert Kostenbewußtsein, Signifikanzorientierung und qualitätsorientiertes Management in der öffentlichen Verwaltung. 
Für alle TZ-Maßnahmen sollten verstärkt lokale Sachverständige bzw. Experten und Expertinnen aus anderen Entwicklungsländern eingesetzt werden, die mit der strukturellen Dysfunktionalität öffentlicher Verwaltungen und den für Entwicklungsländer typischen Restriktionen und Engpässen vertraut sind. Dies ist insbesondere in Ländern Afrikas südlich der Sahara wichtig, wo externe Berater aufgrund der Praxis der Kolonialmächte z.T. diskreditiert sind und die aktuelle "aufgesetzte und kostspielige Expertenwirtschaft" ${ }^{\text {"64 }}$ manchmal mehr Probleme schafft als sie löst. ${ }^{65}$

\section{Finanzielle Zusammenarbeit}

Die Finanzielle Zusammenarbeit (FZ) kann zur Verwaltungsförderung nur begrenzt beitragen, da Investitionen und Warenlieferungen nur selten zur Durchführung von Verwaltungsreformen erforderlich sind. Daß FZ auch im Kontext von Verwaltungsreformen stark nachgefragt wird, liegt u.a. daran, daß sie den Interessen der herrschenden Eliten entspricht, da entsprechende Projekte prestigeträchtig sind und darüber hinaus gute Möglichkeiten für Bereicherung und Begünstigung bieten. Sinnvoll sind FZ-Maßnahmen nur, wenn sie in Verwaltungsreformen und umfassende Unterstützungsmaßnahmen eingebunden sind. So führt die Ausstattung von öffentlichen Verwaltungen mit Computern allein nicht zu Effizienz- und Qualitätssteigerungen. Auch im Rahmen von Dezentralisierungsbemühungen kommt es weniger auf die Finanzierung einzelner kommunaler (Investitions-)Projekte als auf die Schaffung von Einnahmequellen für Kommunen durch entsprechende Strukturveränderungen an.

Wichtig ist vielmehr, daß bei allen FZ-Maßnahmen - wie z.B. Strukturanpasssungskofinanzierungen und Infrastrukturprojekten - eventuelle Verwaltungsreformen berücksichtigt und unterstützt werden. So sollte beispielsweise verstärkt mit dezentralen Verwaltungsstrukturen und privaten (Unter-)Auftragnehmern kooperiert werden.

\section{Finanzierung laufender Kosten}

Ein heikles Thema ist die Finanzierung laufender Kosten, die im Rahmen der deutschen Entwicklungszusammenarbeit bislang mit dem Hinweis auf mangelnde Nachhaltigkeit abgelehnt wurde. Anders als durch die zeitweilige Mitfinanzierung von Personalausgaben (insbesondere von Abfindungen, Mindestlöhnen bzw. -gehältern und Gehaltserhöhungen) läßt sich das Problem der gravierenden Unterbezahlung im öffentlichen Dienst jedoch nicht Manuskript). S. 3.

Vgl. E. Jaycox, Capacity Building..., a.a.O. 
lösen. Die Einkommen im öffentlichen Dienst von Entwicklungsländern Afrikas südlich der Sahara nehmen laufend ab und zwingen die öffentlich Bediensteten damit immer mehr, anderen Erwerbstätigkeiten nachzugehen, Bestechungsgelder anzunehmen und/oder öffentliches Eigentum zu unterschlagen. Finanziell akzeptabel ist eine Tätigkeit im öffentlichen Dienst in vielen Ländern nur noch, wenn gleichzeitig für EZ-Institutionen gearbeitet wird, die für die Arbeit an "ihren" Projekten erhebliche Zulagen bezahlen. Diese weitverbreitete Praxis führt zur

- Schwächung der öffentlichen Verwaltung, indem sie die besten Arbeitskräfte von ihrer regulären Tätigkeit im öffentlichen Dienst abzieht, und zur

- Pervertierung der Entwicklungszusammenarbeit, indem aufwendige EZ-Projekte konzipiert werden, obwohl die Entwicklungsländer eigentlich nur an Fahrzeugen, Material und Zuschüssen interessiert sind, die sie anders nicht finanzieren können.

Die Praxis ergänzender Gehaltszahlungen sollte daher grundsätzlich vermieden werden. Statt dessen sollten Geber bereit sein, Personalkosten in Schlüsselbereichen öffentlicher Verwaltungen in entwicklungsorientierten Ländern unter bestimmten Bedingungen ${ }^{66}$ und zeitlich begrenzt zu übernehmen.

\section{Abschließende Bewertung}

Die Erfahrungen und Erkenntnisse der letzten Jahre im Kontext von Wirtschaftskrise, Strukturanpassung und Entwicklungszusammenarbeit belegen, wie wichtig Maßnahmen zur Überwindung struktureller Dysfunktionalität sind und daß entsprechende Unterstützung, insbesondere für reformorientierte Regierungen und Akteure, im Rahmen der Entwicklungszusammenarbeit unverzichtbar ist.

Der vorliegende Artikel hat gezeigt, welche Rolle dabei Verwaltungsreformen spielen können und wie viele Handlungsmöglichkeiten und Ansatzpunkte für Entwicklungszusammenarbeit es in diesem Bereich gibt. 1995 (im Druck). 
responsiveness, stability and issues of development. In other words, the policy objectives of the decentralization programme were geared towards both political and administrative decentralization. The objectives of the programme have not been realized mainly because of the uneven distribution of power relationships and distribution of tasks between the central government and the District Assemblies (DAs) - local government units. The relationship has, therefore, become inappropriate to the nurturing of independent local government units in Ghana.

The paper examines central-local government relations in Ghana and their implications for relocating government administration. Specifically, it emphasizes the political nature of the relationships and focuses on substantive policies, especially financial issues, such as who raises what amount and who spends it for whose benefit with what resultats. The paper argues that subnational governments, if they should have any political credibility and legitimacy, must have some measure of independence in the level of revenue they raise, the choice of public goods on which to spend it and the capacity to "hire and fire" staff.

An evaluation of the nature of intergovernmental relations in Ghana and the degree of autonomy that should be given to local management of resources will be based on four indicators:

(i) choice of levels;

(ii) choice of decentralized authority;

(iii) choice of tasks to decentralize; and

(iv) choice of device used to decentralize power.

\section{Lean management-Reforms in Developing Countries in Sub-Saharan Africa}

\section{By Hildegard Lingnau}

Public administrations often represent a major obstacle to development in developing countries in Sub-Saharan Africa. The analysis of public administration reforms in Benin and Uganda shows that public administration reforms are both necessary and feasible. For reforms to be successful, they require the commitment of a reform-oriented government and a comprehensive approach to overcome structural dysfunctionality. Reform- and development-oriented governments of developing countries like Uganda give an impressive picture of how imaginative public administration reforms can be implemented effectively. If, in contrast, it is not possible to break the dominance of self-enriching ruling classes and to restrict their power, perceptible improvements have yet to occur, as is the case in Benin. The new public management debate and the lean management reform concept in particular offer important starting points and instruments for necessary reform projects and respective support measures in the framework of development cooperation. 